\title{
Does Early Low-Intensity Aerobic Exercise Hasten Recovery in Adolescents With Sport-Related Concussion?
}

\author{
Ryan D. Henke, Savana M. Kettner, Stephanie M. Jensen, Augustus C.K. Greife, \\ and Christopher J. Durall
}

\begin{abstract}
Clinical Scenario: Low-intensity aerobic exercise (LIAEX) below the threshold of symptom exacerbation has been shown to be superior to rest for resolving prolonged ( $>4 \mathrm{wk}$ ) symptoms following sport-related concussion (SRC), but the effects of LIAEX earlier than 4 weeks after SRC need to be elucidated. Focused Clinical Question: Does LIAEX within the first 4 weeks following SRC hasten symptom resolution? Summary of Key Findings: Two randomized controlled trials (RCT) and 1 nonrandomized trial involving adolescent athletes (10-19 y) were included. One RCT reported faster recovery time with LIAEX versus placebo stretching. Likewise, recovery time was faster with LIAEX versus rest in the nonrandomized trial, but not in the underpowered RCT, although effect sizes were similar between these studies ( 0.5 and 0.4 , respectively). All 3 studies reported a reduction in concussion symptom severity with LIAEX; however, the magnitude of symptom reduction across the recovery timeline was greater in the LIAEX group than the rest group in the nonrandomized trial, but not the 2 RCTs. Importantly, no adverse effects or incidence of delayed recovery from LIAEX were reported in any of the studies. Clinical Bottom Line: LIAEX initiated within 10 days after SRC may facilitate a faster recovery time versus placebo stretching or rest, although additional clinical trials are strongly advised to verify this. Strength of Recommendation: Level $1 \mathrm{~b}$ and $2 \mathrm{~b}$ evidence suggests subsymptom exacerbation LIAEX may decrease Postconcussion Symptom Scale scores and hasten symptom resolution in adolescent athletes following SRC.
\end{abstract}

Keywords: mild traumatic brain injury, active rehabilitation, return to sport, subsymptom exacerbation

\section{Clinical Scenario}

Historically, adolescent athletes with sport-related concussion (SRC) have been advised to abstain from physical activity until asymptomatic, at which point they may begin a graduated return to sport protocol. ${ }^{1}$ Some researchers, however, have proposed that subsymptom exacerbation low-intensity aerobic exercise (LIAEX) may expedite concussion recovery via increased levels of brain-derived neurotrophic factor. ${ }^{2-5}$ Subsymptom exacerbation LIAEX, starting a minimum of 4 weeks after an SRC, has been reported to be more beneficial than rest, ${ }^{6}$ but the effects of LIAEX earlier than 4 weeks need to be elucidated. This review was conducted to determine if evidence supports initiating subsymptom exacerbation LIAEX within the first 4 weeks following SRC. The review was limited to adolescent athletes (aged 10$19 \mathrm{y}$ ), because, historically, this group experiences high rates of SRC and appears to be more vulnerable to prolonged recovery. ${ }^{1,7}$

\section{Focused Clinical Question}

Does subsymptom exacerbation LIAEX within the first 4 weeks following SRC hasten symptom resolution in adolescent athletes?

\section{Summary of Search, "Best Evidence" Appraised, and Key Findings}

- The literature was searched for studies on the effects of early (within $4 \mathrm{wk}$ ) subsymptom exacerbation LIAEX after medically diagnosed SRC in adolescents.

- The search yielded 3 articles ${ }^{2,7,8}$ that fit inclusion/exclusion criteria. One level $1 \mathrm{~b}$ randomized controlled trial (RCT), ${ }^{2}$ and 1 level $2 \mathrm{~b}$ nonrandomized trial $(\mathrm{NRT})^{7}$ compared LIAEX initiated within 10 days after SRC with usual care (ie, rest). A second level $1 \mathrm{~b} \mathrm{RCT}{ }^{8}$ compared LIAEX initiated within 10 days after SRC with placebo stretching.

- Recovery in the studies was defined as symptom resolution per the Postconcussion Symptom Scale (PCSS) and/or examination by a physician.

- One $\mathrm{RCT}^{8}$ found a significant difference in days to recovery favoring the LIAEX group compared with the stretching group. Likewise, the NRT by the same group of investigators showed faster recovery with LIAEX versus usual care (ie, rest). By contrast, the other $\mathrm{RCT}^{2}$ did not find a significant between-group difference in days to recovery, although this study appears to have been underpowered.

- Notably, there were no reports of adverse effects with LIAEX in any of the reviewed studies.

\section{Clinical Bottom Line}

Henke, Kettner, Jensen, Greife, and Durall are with the Doctor of Physical Therapy Program, University of Wisconsin-La Crosse, La Crosse, WI. Durall is also with Physical Therapy Unit, Student Health Center, University of Wisconsin-La Crosse, La Crosse, WI. Henke (rdhenke12@gmail.com) is corresponding author. 
overall recovery time when compared with rest or placebo stretching, although additional data are needed to substantiate this conclusion. Moderately strong (level 1 and 2) evidence from all 3 studies indicates that SRC symptom severity decreased across the study interval in response to LIAEX. In the reviewed studies, no adverse effects were reported when LIAEX was performed 5 to 7 days per week on a stationary bike or treadmill for 10 to 20 minutes at $50 \%$ to $70 \%$ of age-predicted maximal heart rate ${ }^{2}$ or at $80 \%$ of symptom-threshold heart rate. ${ }^{7,8}$

\section{Strength of Recommendation}

Level 1 evidence from 2 RCTs and level 2 evidence from 1 NRT support early $(<10 \mathrm{~d})$ subsymptom exacerbation LIAEX for adolescents with SRC. It is important to acknowledge that the RCT by Micay et $\mathrm{al}^{2}$ in this review appears to have been underpowered with a total sample size of 15. This small sample may have prevented detection of between-group differences. The NRT by Leddy et $\mathrm{al}^{8,9}$ compared convenience sample data from 2 different published studies, and thus group assignment was not random. Finally, the RCT by Leddy et $\mathrm{al}^{8}$ did not a have a true control group, rather, a placebo stretching group that was compared with the LIAEX group. Given the limited number of studies on this topic and the methodological shortcomings described earlier, additional data from studies with greater methodological quality and homogeneity are advised before widespread adoption should be advocated.

\section{Search Strategy}

\section{Terms Used to Guide Search Strategy}

- Patient/Client group: (adolescents OR athletes OR youth) AND (concussion OR traumatic brain injury OR mTBI)

- Intervention/Assessment: (symptom*OR sub-symptom*OR symptom threshold) AND (graded exercise program OR aerobic OR active rehab*)

- Comparison: rest OR no activity

- Outcomes: time OR duration OR symptom resolution OR symptom relief OR return to sport

\section{Sources of Evidence Searched}

- CINAHL Plus with Full Text

- Cochrane Central Register of Controlled Trials

- Cochrane Clinical Answers

- Cochrane Database of Systematic Reviews

- MEDLINE

- MEDLINE with Full Text

- SPORTDiscus with Full Text

\section{Inclusion and Exclusion Criteria}

\section{Inclusion}

- Limited to human subjects 10-19 years old

- Aerobic exercise initiated within 4 weeks after SRC diagnosis

- Published in English language

\section{Exclusion}

- Aerobic exercise initiated $\geq 4$ weeks after SRC

\section{Results of Search}

The initial search in October 2018 yielded 24 studies that were reviewed for appropriateness based on inclusion and exclusion criteria. Of these, 2 articles $^{2,7}$ were selected for critical appraisal. An additional article, published in February 2019, was identified on PubMed. ${ }^{8}$ A summary of the selected articles is provided in Tables 1 and 2 .

\section{Best Evidence}

The studies in Tables 1 and 2 were identified as the best available evidence for this review. Two studies were level $1 \mathrm{~b}$ evidence RCT and 1 study was a level $2 \mathrm{~b}$ evidence NRT. Levels of evidence for this review were based on the Centre for Evidence-Based Medicine 2009 criteria. $^{10}$

\section{Implications for Practice, Education, and Future Research}

Using LIAEX after SRC is a relatively recent practice, and, accordingly, the safety and efficacy of this approach need to be well established by high-quality research before widespread clinical adoption should be considered. The reviewed studies reported no adverse effects in symptomatic adolescents following subsymptom exacerbation LIAEX started within 10 days post-SRC. In the NRT, none of the 24 participants in the LIAEX group had delayed recovery, while 4 of the $30(13 \%)$ rest group participants had delayed recovery. ${ }^{7}$ In addition, in the RCT by Leddy et al, ${ }^{8} 2$ of the $52(3.8 \%)$ participants with acute SRC in the LIAEX group had delayed recovery, while 7 of the 51 (13.7\%) stretching group participants had delayed recovery. Therefore, a potentially important advantage of exercise-based treatment early in the SRC recovery process may be the reduced incidence of delayed recovery.

Regarding the efficacy of subsymptom exacerbation LIAEX for concussed adolescents, all 3 studies reported a reduction in SRC symptom severity across their study intervals. The magnitude of symptom reduction across the recovery timeline was greater in the LIAEX group than the rest group in the NRT, ${ }^{7}$ but not the 2 RCTs. $^{2,8}$ Reviewers calculated effect sizes for between-group symptom severity reduction (via PCSS scores) from the data provided in the Micay et $\mathrm{al}^{2} \mathrm{RCT}$; effect sizes were considered clinically significant $(2.8$ between weeks 1 and $3 ; 1.6$ between

\section{Table 1 Summary of Study Designs of Retrieved Articles}

\begin{tabular}{lccc}
\hline $\begin{array}{l}\text { Level of } \\
\text { evidence }\end{array}$ & $\begin{array}{l}\text { Study design/ } \\
\text { methodology }\end{array}$ & $\begin{array}{c}\text { Number } \\
\text { located }\end{array}$ & Study \\
\hline $1 \mathrm{~b}$ & RCT & 2 & $\begin{array}{l}\text { Micay et } \mathrm{al}^{2} \\
\text { Leddy et } \mathrm{al}^{8}\end{array}$ \\
$2 \mathrm{~b}$ & Quasi-experimental NRT & 1 & ${\text { Leddy et } \mathrm{al}^{7}}^{\text {Ledd }}$
\end{tabular}

Abbreviations: NRT, nonrandomized trial; RCT, randomized controlled trial. 
Table 2 Summary of Best Evidence

\begin{tabular}{|c|c|c|c|}
\hline & Micay et $\mathrm{al}^{2}$ & Leddy et $\mathrm{al}^{7}$ & Leddy et $\mathrm{al}^{8}$ \\
\hline Study design & Randomized controlled trial & Nonrandomized trial & Randomized controlled trial \\
\hline \multirow[t]{3}{*}{ Participants } & $\begin{array}{l}15 \text { male athletes diagnosed with SRC } \\
\text { and symptomatic at day } 5 \text { postinjury } \\
\text { were randomized into EXG or UCG. }\end{array}$ & $\begin{array}{l}54 \text { male athletes diagnosed with SRC } \\
1-9 \text { d prior to study enrollment. Data for } \\
\text { EXG were extracted from another ran- } \\
\text { domized controlled trial on subthreshold } \\
\text { aerobic exercise prescription. }{ }^{8}\end{array}$ & $\begin{array}{l}103 \text { athletes }(\mathrm{F}=48, \mathrm{M}=55) \text { diagnosed } \\
\text { with SRC } 1-9 \mathrm{~d} \text { prior to study } \\
\text { enrollment were randomized into } \\
\text { EXG or placebo stretching group. } \\
\text { No interventions were started earlier } \\
\text { than } 48 \mathrm{~h} \text { postinjury. }\end{array}$ \\
\hline & Age $=14-18 \mathrm{y}$, mean age $=15 \mathrm{y}$ & Age $=13-18 \mathrm{y}$, mean age $=15 \mathrm{y}$ & Age $=13-18 \mathrm{y}$, mean age $=15 \mathrm{y}$ \\
\hline & $\begin{array}{l}\text { Demographic variables did not differ } \\
\text { between groups at the start of the study. }\end{array}$ & $\begin{array}{l}\text { Demographic variables did not differ } \\
\text { between groups at the start of the study. }\end{array}$ & $\begin{array}{l}\text { Demographic variables did not differ } \\
\text { between groups at the start of the study. }\end{array}$ \\
\hline $\begin{array}{l}\text { Intervention } \\
\text { investigated }\end{array}$ & $\begin{array}{l}\text { EXG }(\mathrm{n}=8) \text { participated in } 8 \text { sessions } \\
\text { of supervised aerobic exercise on a sta- } \\
\text { tionary cycle ergometer wearing an HR } \\
\text { monitor. Sessions started on day } 6 \\
\text { postinjury. Each session included } \\
\text { a } 5 \text {-min warm-up and } 5 \text {-min cooldown. } \\
\text { Session } 1 \text { was } 10 \text { min in duration at } 50 \% \\
\text { of age-predicted } \mathrm{HR}_{\max } \text {. Session } 2 \text { was } \\
20 \text { min at } 50 \% \mathrm{HR}_{\max } \text {. Subsequent } \\
\text { sessions were } 20 \text { min in duration, } \\
\text { but intensity was increased by } 5 \% \text { of } \\
\mathrm{HR}_{\text {max }} \text { until } 70 \% \mathrm{HR}_{\max } \text { was achieved. } \\
\text { Participants exercised at this intensity } \\
\text { during all subsequent sessions. Exercise } \\
\text { sessions occurred on } 2 \text { consecutive }\end{array}$ & $\begin{array}{l}\text { EXG }(\mathrm{n}=24) \text { participated in daily } \\
\text { supervised } 20 \text {-min aerobic exercise } \\
\text { sessions on a treadmill or stationary bike } \\
\text { at } 80 \% \text { subthreshold HR wearing an HR } \\
\text { monitor. Sessions started within } 9 \mathrm{~d} \\
\text { postinjury. Each session included a } \\
\text { 5-min warm-up and 5- to } 10 \text {-min } \\
\text { cooldown. Prior to first exercise session, } \\
\text { the BCTT was used to determine HR } \\
\text { at symptom exacerbation ( } \geq 3 \text {-point } \\
\text { increase on VAS) or voluntary fatigue } \\
\text { ( } \geq 17 \text { RPE). Participants were instructed } \\
\text { to stop exercising if symptom } \\
\text { exacerbation occurred during daily } \\
\text { exercise sessions. }\end{array}$ & $\begin{array}{l}\text { EXG }(\mathrm{n}=52) \text { participated in } 20 \text {-min } \\
\text { daily aerobic exercise on a treadmill or } \\
\text { stationary bike at } 80 \% \text { subthreshold HR } \\
\text { wearing an HR monitor. Supervised } \\
\text { LIAEX was performed at home or in } \\
\text { a gym. Sessions started within } 9 \mathrm{~d} \\
\text { postinjury. Prior to first exercise session, } \\
\text { the BCTT was used to determine HR } \\
\text { at symptom exacerbation. Participants } \\
\text { were instructed to stop exercising if } \\
\text { symptom exacerbation occurred during } \\
\text { exercise sessions at home ( } \geq 2 \text {-point } \\
\text { increase on VAS). Participants com- } \\
\text { pleted a weekly BCTT to calculate } \\
\text { a new target HR. }\end{array}$ \\
\hline
\end{tabular}

Control The UCG $(n=7)$ were progressed through a 6-stage progression of activity protocol (per Berlin 2016 Guidelines), under the direction of their sports medicine physician.

Outcome measure(s)

Primary findings

Secondary findings

\section{No participants experienced symptom} exacerbation during intervention; participants completed all intervention sessions.

Symptom severity and exacerbation per PCSS.

Time (in days) to medical clearance.

No significant difference between groups in average time to medical clearance $(P=.87$; Cohen $d=0.4)$. PCSS scores significantly decreased in both groups, although differences between groups were not significant. Large between-groups effect sizes favored EXG for reducing PCSS scores (effect sizes were calculated, not reported): (1) Weeks 1-3 (Cohen $d=2.8$ ) (2) Weeks $1-4$ (Cohen $d=1.6$ )

exacerbation during intervention;
intervention sessions.

Level of evidence $1 \mathrm{~b}$

Validity score
PEDro score: $6 / 10$
The UCG $(n=30)$ was a subset from a previously published study. ${ }^{9}$ Participants were instructed to follow standard care model for return to sport/ school. Activity levels were not strictly monitored.

Time (in days) to recovery from initial visit.

Return to baseline symptoms per PCSS.

Recovery time from initial visit was significantly faster for EXG than UCG $(P=.048)$ with a moderate effect size (calculated Cohen $d=0.5$ ) favoring EXG.

Percentage of participants not recovered (total, physical, cognitive, and sleep symptoms) by day 14 was significantly different between groups favoring EXG.

No EXG participants experienced prolonged recovery ( $>30 \mathrm{~d}$ ) vs $4 / 30(13 \%)$ in the EXG had prolonged recovery.

$2 \mathrm{~b}$

PEDro score: $4 / 10$
The stretching group $(\mathrm{n}=51)$ was instructed to follow a gentle, whole-body stretching program that was not intended to noticeably raise HR. Stretching was progressed each week. Participants completed a weekly BCTT during clinic visits.

Time (in days) to recovery since date of injury.

Return to baseline symptoms per PCSS.

EXG recovered significantly faster than stretching group $(z=2.82, P=.01)$. EXG recovered in a median of $13 \mathrm{vs} 17 \mathrm{~d}$ in stretching group $(P=.01)$.

Total self-reported symptom scores appeared to decrease more rapidly in the EXG. Decreases in symptom scores for the EXG achieved significance on days 5,12 , and 18 , but not for the other $18 \mathrm{~d}$ of the study.

Seven participants in the stretching group experienced delayed recovery since injury ( $>30 \mathrm{~d}$ ) compared with 2 participants in the EXG; this was not found to be significant $(P=.08)$ $1 \mathrm{~b}$

PEDro score: $5 / 10$ 


\section{Table 2 (continued)}

\begin{tabular}{|c|c|c|c|}
\hline & Micay et $\mathrm{al}^{2}$ & Leddy et $\mathrm{al}^{7}$ & Leddy et $a^{8}$ \\
\hline Conclusion & $\begin{array}{l}\text { In male adolescents diagnosed with SRC } \\
\text { and symptomatic after } 5 \mathrm{~d} \text {, both LIAEX } \\
\text { and rest were beneficial in decreasing } \\
\text { symptom severity. LIAEX was not } \\
\text { associated with a faster recovery time } \\
\text { than rest, although the small sample may } \\
\text { have hindered detection of between- } \\
\text { group differences. Aerobic exercise did } \\
\text { not cause symptom exacerbation and } \\
\text { thus appears to be safe and feasible in } \\
\text { potentially aiding symptom resolution. }\end{array}$ & $\begin{array}{l}\text { Following SRC, an early individualized } \\
\text { subthreshold LIAEX intervention may } \\
\text { safely hasten symptom resolution in } \\
\text { adolescent males. Accordingly, sub- } \\
\text { threshold LIAEX within } 10 \mathrm{~d} \text { following } \\
\text { injury may reduce the number of patients } \\
\text { who experience prolonged recovery. }\end{array}$ & $\begin{array}{l}\text { Use of individualized subsymptom } \\
\text { threshold aerobic exercise within the first } \\
\text { week following SRC may safely speed } \\
\text { recovery in male and female adolescents. } \\
\text { Reduced incidence of delayed recovery } \\
\text { appears to be the primary benefit of early } \\
\text { subthreshold exercise treatment. }\end{array}$ \\
\hline
\end{tabular}

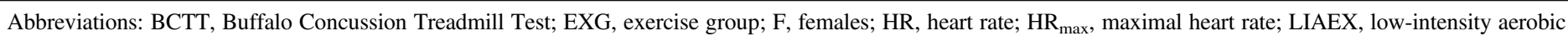
exercise; M, males; PCSS, Postconcussion Symptom Scale; RPE, rating of perceived exertion; SRC, sport-related concussion; UCG, usual care group; VAS, visual analog scale.

weeks 1 and 4), favoring the LIAEX group. In the NRT, adolescents who started LIAEX within 9 days of SRC had greater symptom reduction across the study period than the usual care (ie, rest) group. They also reported the LIAEX group had a significantly shorter recovery time than the usual care group, although their control data set was imported from another study, and group assignment was not randomized. ${ }^{7}$ By contrast, the RCT by Micay et al did not detect between-group differences in recovery time, although their small sample size $(\mathrm{N}=15)$ may have lacked the statistical power to detect differences. According to Micay et $a{ }^{2}{ }^{2}$ a calculated sample size of 30 would be needed to detect between-group differences with an effect size of 0.8. Calculated effect sizes for reduction in time to medical clearance were moderate in both the Micay et $\mathrm{al}^{2}$ and Leddy et $\mathrm{al}^{7}$ studies $(0.4$ and 0.5 , respectively). In the RCT by Leddy et al, ${ }^{8}$ both groups reported decreases in PCSS scores through the 21 days of the study; however, when compared with the stretching group, the LIAEX group reported significant PCSS score decreases on days 5, 12, and 18. Additional RCTs are needed to definitively answer the clinical question regarding the impact of subsymptom exacerbation LIAEX on overall SRC recovery time in this population, although the initial data are encouraging.

Clinicians should educate patients that aerobic exercise is beneficial for the human brain and that subsymptom exacerbation LIAEX does not appear to delay recovery, but it may speed recovery in adolescents with acute postconcussive symptoms. This seems concordant with a recent international consensus statement that recommends a more active approach to SRC treatment. ${ }^{1}$ There is evidence that aerobic exercise can promote neuron growth and repair after concussions through increased levels of brain-derived neurotrophic factor. ${ }^{5}$ Levels of brain-derived neurotrophic factor have been shown to increase in healthy athletes immediately after cycle ergometry to exhaustion ${ }^{11}$ and in rodents that initiated exercise within 1 to 3 days after concussion. ${ }^{12}$ In the later study, concussed rodents that were exercised also had significant improvements in motor and cognitive functioning. Current evidence points to inferior neurocognitive performance of athletes engaging in high levels of activity following SRC when compared with athletes engaging in moderate levels of activity. ${ }^{13}$ Given this, clinicians should emphasize to their patients that any exercise or activity should be below the threshold of symptom exacerbation.

Future research on this topic would be strengthened by: (1) using mixed-gender participant groups, (2) including a control group and random group assignment for interventional comparisons, (3) determining optimal sample sizes a priori via a power analysis, (4) employing a standardized LIAEX protocol, (5) using standardized outcomes measures to determine readiness to return to sport/activity (ie, medical clearance), and (6) reporting effect sizes so the clinical significance can be appreciated. The RCT by Micay et $\mathrm{al}^{2}$ and the NRT by Leddy et $\mathrm{al}^{7}$ included male subjects only for convenience.' Females, however, may be more likely to have a delayed recovery after SRC. ${ }^{14}$ As with the reviewed RCT by Leddy et $\mathrm{al}^{8}{ }^{8}$ inclusion of both males and females in future studies will improve generalizability. The consistent utilization of randomly assigned control or comparator groups will aid in estimating the influence of aerobic exercise post-SRC. In addition, sample size calculations should be performed to ensure studies on this topic are adequately powered.

There is a need to determine optimal exercise protocols that could be adopted by future researchers. Two of the studies in this review utilized daily subthreshold aerobic exercise lasting 20 to 30 minutes, ${ }^{7,8}$ while the third study used a prescribed progressive aerobic exercise program, based on age-predicted maximal heart rate, beginning at 10 minutes and increasing over time to 20 minutes. $^{2}$ In both articles by Leddy et al, ${ }^{7,8}$ the heart rate at symptom exacerbation was used to determine a target subsymptom threshold heart rate for aerobic exercise. Determination of optimal LIAEX dosage is needed, as 2 studies implemented daily exercise $^{7,8}$ and the other prescribed exercise 5 days per week. ${ }^{2}$ Ongoing research to develop a standardized aerobic exercise protocol for concussed patients is being conducted. ${ }^{15}$ Future researchers should aim to standardize outcome measures; all 3 of the reviewed studies included the PCSS as an outcome measure, although this is a highly subjective, symptom-based test. Furthermore, in the studies by Leddy et al, ${ }^{7,8}$ symptom recovery was defined as a PCSS score of $<7$ for 3 consecutive days, while Micay et $\mathrm{al}^{2}$ relied solely on physician examination to determine recovery. ${ }^{2}$ Thus, there is a need for more standardization in determining recovery and readiness for return to sport after SRC. As with many health care issues, greater methodological consistency in future studies should improve confidence in the validity of results and enhance clinical decision making.

The results of this review suggest subsymptom exacerbation LIAEX administered within 10 days following SRC in adolescents does not appear to delay recovery and may result in greater symptom reduction and faster medical clearance than rest. Clinicians who consider applying the LIAEX protocols, as described in the clinical bottom line, should emphasize to their patients that any exercise or activity should be below the threshold of symptom exacerbation. Information in this critically appraised topic should 
be reviewed in 3 years or after the publication of high-quality RCTs on this topic to determine if the recommendations suggested earlier should be modified.

\section{Acknowledgment}

The authors have no conflicts of interest or sources of funding related to the topic or production of this manuscript.

\section{References}

1. McCrory P, Meeuwisse W, Dvorak J, et al. Consensus statement on concussion in sport - the 5th international conference on concussion in sport held in Berlin, October 2016. Br J Sports Med. 2017;51(11): 838-847. PubMed ID: 28446457

2. Micay R, Richards D, Hutchison MG. Feasibility of a postacute structured aerobic exercise intervention following sport concussion in symptomatic adolescents: a randomised controlled study. BMJ Open Sport Exerc Med. 2018;4:e000404. PubMed ID: 30018795 doi:10. 1136/bmjsem-2018-000404

3. Carter JB, Banister EW, Blaber AP. Effect of endurance exercise on autonomic control of heart rate. Sports Med. 2003;33:33-46. PubMed ID: 12477376 doi:10.2165/00007256-200333010-00003

4. Doering TJ, Resch KL, Steuernagel B, Brix J, Schneider B, Fischer GC. Passive and active exercises increase cerebral blood flow velocity in young, healthy individuals. Am J Phys Med Rehabil. 1998;77:490-493. PubMed ID: 9862534 doi:10.1097/00002060-199811000-00006

5. Erickson KI, Voss MW, Prakash RS, et al. Exercise training increases size of hippocampus and improves memory. Proc Natl Acad Sci USA. 2011;108(7):3017-3022. PubMed ID: 21282661 doi:10.1073/pnas. 1015950108

6. Ritter KG, Hussey MJ, Valovich McLeod TC. Subsymptomatic aerobic exercise for patients with postconcussion syndrome: a critically appraised topic. J Sport Rehabil. 2019;28(2):211-216. PubMed ID: 28952858 doi:10.1123/jsr.2017-0159

7. Leddy JJ, Haider MN, Hinds AL, Darling S, Willer BS. A preliminary study of the effect of early aerobic exercise treatment for sportrelated concussion in males [published online ahead of print
September 19, 2018]. Clin J Sport Med. doi:10.1097/JSM. 0000000000000663

8. Leddy JJ, Haider MN, Ellis MJ, et al. Early subthreshold aerobic exercise for sport-related concussion: a randomized clinical trial. JAMA Pediatr. 2019;173(4):319-325; doi:10.1001/jamapediatrics. 2018.4397

9. Leddy JJ, Hinds AL, Miecznikowski J, et al. Safety and prognostic utility of provocative exercise testing in acutely concussed adolescents: a randomized trial. Clin J Sport Med. 2018;28: 13-20. PubMed ID: 29257777 doi:10.1097/JSM.000000 0000000431

10. Oxford Centre for Evidence-Based Medicine. OCEBM levels of evidence. https://www.cebm.net/2009/06/oxford-centre-evidencebased-medicine-levels-evidence-march-2009/. Published 1998. Accessed September 2018.

11. Rojas VS, Struder HK, Vera Wahrmann B, Schmidt A, Bloch W, Hollmann W. Acute BDNF and cortisol response to low intensity exercise and following ramp incremental exercise to exhaustion in humans. Brain Res. 2006;1121:59-65. doi:10.1016/j.brainres.2006. 08.105

12. Mychasiuk R, Hehar H, Ma I, Candy S, Esser MJ. Reducing the time interval between concussion and voluntary exercise restores motor impairment, short-term memory, and alterations to gene expression. Eur J Neurosci. 2016;44:2407-2417. PubMed ID: 27521273 doi:10. 1111/ejn. 13360

13. Majerske CW, Mihalik JP, Ren D, et al. Concussion in sports: postconcussive activity levels, symptoms, and neurocognitive performance. J Athl Train. 2008;43(3):265-274. PubMed ID: 18523563 doi:10.4085/1062-6050-43.3.265

14. Iverson GL, Gardner AJ, Terry DP, et al. Predictors of clinical recovery from concussion: a systematic review. $\mathrm{Br} J$ Sports Med. 2017;51:941-948. PubMed ID: 28566342 doi:10.1136/bjsports2017-097729

15. Ledoux AA, Barrowman NJ, Boutis K, et al. Multicentre, randomised clinical trial of paediatric concussion assessment of rest and exertion (PedCARE): a study to determine when to resume physical activities following concussion in children. Br J Sports Med. 2019;53(3):195. PubMed ID: 28701360 doi:10.1136/bjsports2017-097981 